\title{
The normal structure and function of CD44 and its role in neoplasia
}

\author{
R J S Sneath, D C Mangham
}

\begin{abstract}
CD44 is a transmembrane glycoprotein, the variant isoforms of which are coded for by alternative splicing, with the most prolific isoform being CD44 standard. CD44 is found in a wide variety of tissues including the central nervous system, lung, epidermis, liver, and pancreas, whereas variant isoforms of CD44 (CD44v) appear to have a much more restricted distribution. Variants of CD44 are expressed in tissues during development, including embryonic epithelia. Known functions of CD44 are cellular adhesion (aggregation and migration), hyaluronate degradation, lymphocyte activation, lymph node homing, myelopoiesis and lymphopoiesis, angiogenesis, and release of cytokines. The functions of CD44 are principally dependant on cellular adhesion in one setting or another. The role of CD44 in neoplasia is less well defined, although metastatic potential can be conferred on non-metastasising cell lines by transfection with a variant of CD44 and high levels of CD44 are associated with several types of malignant tumours. The physiological functions of CD44 indicate that the molecule could be involved in the metastatic spread of tumours. Many studies have investigated the pattern of CD44 distribution in tumours and some observations suggest that certain cells do not use CD44 in tumorigenesis or in the production of metastases. However, the data are extremely conflicting, and further studies are needed to establish the prognostic value of CD44 and its variant isoforms. The precise function of CD44 in the metastatic process and the degree of involvement in human malignancies has yet to be established fully. (f Clin Pathol: Mol Pathol 1998;51:191-200)
\end{abstract}

Keywords: CD44; variant isoforms; cellular adhesion; tumorigenesis; metastasis

Pathology

Department, The

University of

Birmingham B15 2TT,

UK

R J S Sneath

Correspondence to: Dr DC Mangham, Department of

Musculoskeletal Pathology,

The Royal Orthopaedic

Hospital, 111 Dale Road,

Selly Oak, Birmingham B29 6AY, UK.

Accepted for publication 14 April 1998 that a variety of carcinoma cell lines and solid tumours expressed the CD44 gene. In 1991, Günthert and colleagues ${ }^{2}$ discovered that an isoform of CD44 when inserted into the genetic sequence of a non-metastasising tumour gave it metastatic properties. These initial discoveries indicating that CD44 was involved in the metastatic process led to a large amount of research into the possible mechanisms and the degree of involvement of CD44.

\section{Background}

As with most clusters of differentiation antigens, several centres discovered the protein individually and each ascribed their own name to it. Previous names for CD44 include:

- Pgp-1 (Hughes and colleagues, ${ }^{3}$ Trowbridge and colleagues $\left.{ }^{4}\right)$

- ECMRIII (Carter and Wayner, ${ }^{5}$ Gallatin and colleagues ${ }^{6}$ )

- Hermes antigen (Jalkanen and colleagues ${ }^{7}$ )

- Ly-24, H-CAM and gp90 $0^{\text {Hermes }}$ (Haynes and colleagues $^{89}$ ).

Sequencing studies and the use of monoclonal antibodies have revealed that all these molecules are identical. The International Workshop on Human Leukocyte Differentiation Antigens have ascribed the CD number 44 to the protein.

\section{Structure and isoforms}

CD44 is a glycoprotein encoded on the short arm of chromosome $11 .^{10}$ The genetic sequence can code for a variety of different proteins by selecting certain exons within the sequence, that is, the gene contains constant and variable exons. Variation occurs at the transcription stage and the splicing together of variant exons to form mRNA is called alternative splicing.

The mechanism that controls alternative splicing is unknown, although there are reports of regulation of the CD44 promoter by the oncogene ras. ${ }^{11}{ }^{12}$ Individual cells have the ability to change the pattern of their CD44 expression according to their needs.

The most prolific isoform of CD44 has been termed CD44 standard (CD44s) and is a protein of $37 \mathrm{kDa}$. The mRNA of this isoform contains no variant exons. There are a total of nine variant regions that can be coded for, $\mathrm{v} 2-\mathrm{v} 10$ ( $\mathrm{vl}$ was isolated in rats and is not present in human DNA). Variant CD44 $(\mathrm{CD} 44 \mathrm{v})$ can contain one or more variant regions, such as CD44v6 or CD44v3-v7. Many combinations of these variant exons are possible and individual cells can repeatedly change the splicing of CD44 pre-mRNA, giving the potential for great diversity. Using polymerase chain reaction (PCR) analysis, over 30 different splice products have been detected to date, ${ }^{13}$ and sequence diversity has been said to exceed that of any other known protein family created by alternative splicing. 
Extracellular

Constant

exon number $\quad \begin{array}{lllll}1 & 2 & 3 & 4 & 5\end{array}$

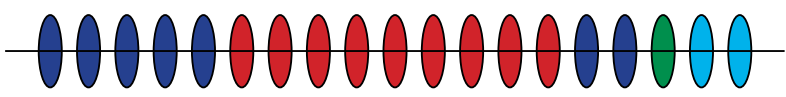

Variable Exon No.

v2 v3 v4 v5 v6 v7 v8 v9 v10

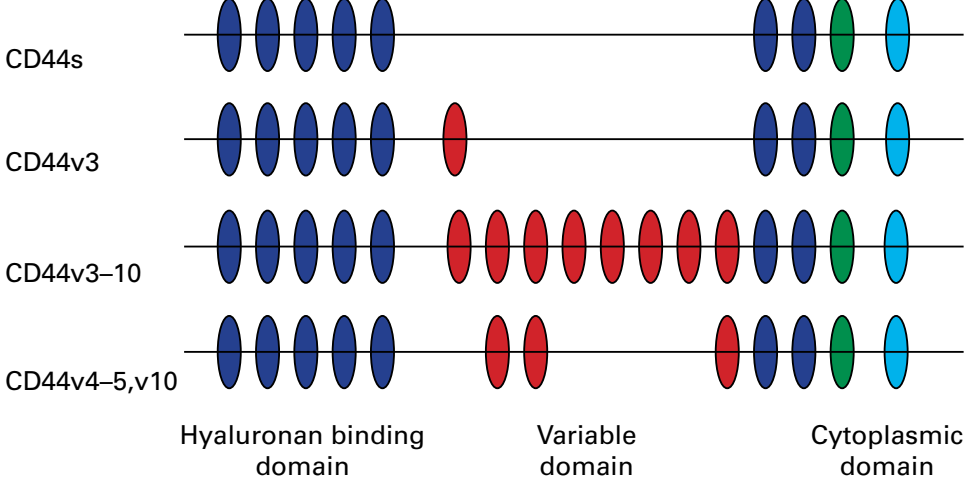

Figure 1 The genomic structure of CD44 (top) with examples of potential isoforms of CD44. TM, transmembrane.

The molecular weight of the standard 37 $\mathrm{kDa}$ protein core is increased to $80-100 \mathrm{kDa}$ as a result of glycosaminoglycan side chain attachment. Glycosaminoglycans are repeating disaccharide units with amino linkage groups that attach the chain to their core proteins. Examples of these side chains include heparan sulphate and chondroitin sulphate. These are large molecules with highly charged sulphate and carboxylate groups. The varying degree of glycosylation can affect some of the functions of the CD44 molecule. The negatively charged environment produced by these chains attracts positive ions and so creates an osmotic potential. Differing CD44 isoforms appear to have characteristic patterns of glycosylation. ${ }^{12}$ CD44s has a minor fraction of chondroitin sulphate, whereas the keratinocyte CD44 molecule (CD44v3-v10) is glycosylated principally by heparan sulphate. There are isoforms of CD44 that occur in both the glycosylated and non-glycosylated forms, suggesting that some of the molecule's functions are not dependant on the glycosaminoglycan side chains. ${ }^{14}$ Some isoforms of CD44 exceed $200 \mathrm{kDa}$ as a result of their degree of glycosylation. Isoforms of intermittent size (110-160 kDa) include forms expressed primarily on epithelial cells. ${ }^{15}$

CD44 is a transmembrane molecule, most of which is sited extracellularly. ${ }^{16}$ The carboxyterminal end of the protein chain is intracellular and forms part of the cytoplasmic domain that is encoded by exon 10 or, alternatively, exon $9^{17}$ (fig 1 ). The transmembrane segment is also encoded by one exon (exon 8 ). The extracellular part of the molecule consists of the middle (variable) domain and the aminoterminal (hyaluronan binding) domain. The variable domain is where differing isoforms express their characteristic variant protein, encoded by the variant exons v2-v10 (between exon 5 and exon 6).
The cytoplasmic domain interacts with the cytoskeleton of the cell. ${ }^{18}$ The degree of and nature of this interaction are, as yet, not fully understood. Neame and colleagues ${ }^{19}$ showed that the loss of the cytoplasmic tail did not alter many of the functions of CD44 and proposed a putative linker molecule interacting with the rest of the CD44 molecule. However, the cytoplasmic domain is required for distributing CD44 to specific areas of the cell ${ }^{20}$ and also increases the longevity of the molecule. The cytoplasmic tail also influences the binding of CD44 with hyaluronate, the molecule's principal ligand. ${ }^{21}$

CD44 is able to crosslink on the cell membrane, and the pattern of this crosslinking varies between CD44s and CD44v. ${ }^{20}{ }^{21}$ Only $\mathrm{CD} 44 \mathrm{v}$ can form oligomers, the molecules involved all being of the same isoform (homooligomerisation). Hyaluronan binding can be influenced by this crosslinking of CD $44 .^{20}$

\section{Distribution of CD44 in normal tissues} ADULT TISSUES

CD44s, as its name implies, was first isolated on haemopoietic cells. ${ }^{22}$ It has since been found on a wide range of tissues including the central nervous system, lung, epidermis, liver, and pancreas (table 1) ${ }^{23-25} \mathrm{CD} 44 \mathrm{v}$ appears to have a much more restricted distribution and is expressed on a variety of epithelial cells in a tissue specific pattern, suggesting that the process of alternative splicing is normally tightly regulated. This also suggests that these isoforms, with a specific exon sequence and restricted distribution, have different, additional functions to CD $44 \mathrm{~s}^{26}$ Examples of cells expressing CD 44v include keratinocytes, activated lymphocytes, macrophages, and some epithelial cells on the bladder, stomach, and uterine cervix. ${ }^{27}$

As the table indicates, squamous epithelium has a high level of CD44s and CD44v expression. Keratinocytes express CD44s and at least five isoforms containing different numbers of variable exons including v2-v10, v3-v10, $\mathrm{v} 4-\mathrm{v} 10$, v6-v10, and v8-v10. ${ }^{15}{ }^{28}$ In normal human keratinocytes, the main CD44 isoform, v3-v10, has both heparan sulphate and chondroitin sulphate side chains. ${ }^{15}{ }^{28}$ Squamous epithelium has also been seen to express intron 9. ${ }^{23}$

CD44 expression within an epithelial tissue can vary between layers and also between cells. Dall and colleagues ${ }^{29}$ reported that CD44v6 expression was limited to the stratum basale and the stratum spinosum of normal uterine cervical squamous epithelium. Korabiowska and colleagues $^{30}$ reported that CD44 was not expressed in normal melanocytes and naevi, although the surrounding keratinocytes express CD44v3-10.

\section{Fetal tissues}

Numerous variants of CD44 have also been found to be expressed strongly on tissues during stages of development, including expression on embryonic epithelia. ${ }^{31}$ In the embryonic mouse, Yu and colleagues ${ }^{32}$ demonstrated variant expression in actively proliferating and 
Table 1 Differential expression of CD44 variants in normal human tissue ${ }^{23}$

\begin{tabular}{|c|c|c|c|c|c|c|c|c|}
\hline Tissue & $C D 44 s$ & $C D 44 v 6$ & $C D 44 v 7$ & $C D 44 v 8$ & $\begin{array}{l}C D 44 v 7 \\
\text { and v8 }\end{array}$ & $\begin{array}{l}C D 44 v 8 \\
\text { and v9 }\end{array}$ & $\begin{array}{l}C D 44 v 7, \\
\text { v8 and v9 }\end{array}$ & Intron 9 \\
\hline Skin & +++ & +++ & +++ & +++ & +++ & +++ & +++ & ++ \\
\hline Gingiva & +++ & +++ & +++ & +++ & +++ & +++ & +++ & +++ \\
\hline Tongue & +++ & +++ & +++ & +++ & +++ & +++ & +++ & +++ \\
\hline Larynx & +++ & +++ & +++ & +++ & +++ & +++ & +++ & +++ \\
\hline Oesophagus & +++ & +++ & +++ & +++ & +++ & +++ & +++ & +++ \\
\hline Bronchus & ++ & ++ & ++ & ++ & ++ & ++ & ++ & + \\
\hline Lung & +++ & ++ & ++ & ++ & ++ & ++ & ++ & + \\
\hline Stomach & +++ & + & + & + & ++ & ++ & ++ & - \\
\hline Duodenum & +++ & + & + & + & ++ & ++ & ++ & - \\
\hline Jejunum & +++ & + & + & + & ++ & ++ & ++ & - \\
\hline Ileum & +++ & + & + & + & ++ & ++ & ++ & - \\
\hline Colon & +++ & $+/++$ & $+/++$ & $+/++$ & ++ & ++ & ++ & $-1+$ \\
\hline Rectum & +++ & $+/++$ & $+/++$ & $+/++$ & ++ & ++ & ++ & $-1+$ \\
\hline Parotid gland & +++ & ++ & ++ & ++ & +++ & +++ & +++ & + \\
\hline Submandibular gland & +++ & ++ & ++ & ++ & +++ & +++ & +++ & + \\
\hline Thyroid gland & + & + & + & + & ++ & +++ & ++ & - \\
\hline Pancreas & ++ & ++ & ++ & ++ & +++ & +++ & +++ & + \\
\hline Adrenal gland & - & - & - & - & + & + & + & - \\
\hline Ovary & - & - & - & - & + & + & + & - \\
\hline Myometrium & +++ & - & - & - & + & + & + & - \\
\hline Cervix & +++ & +++ & +++ & +++ & +++ & +++ & +++ & +++ \\
\hline Liver & - & - & - & - & - & - & - & - \\
\hline Bile duct & - & - & - & - & - & - & - & - \\
\hline Kidney, cortex & + & - & + & + & + & + & + & - \\
\hline Ureter & + & - & - & - & - & - & - & - \\
\hline Bladder & +++ & ++ & ++ & +++ & +++ & +++ & +++ & ++ \\
\hline Spleen & ++ & ++ & ++ & ++ & ++ & ++ & ++ & - \\
\hline Lymphocytes & +++ & - & - & - & - & - & - & - \\
\hline
\end{tabular}

Expression: +++, strong; ++, moderate; +, weak; -, negative.

invaginating epithelia at sites of epithelialmesenchymal interaction. The most prominent of these variants were CD44v3-v10, CD44v4v10, and CD44v6-v10.

\section{Physiological roles of CD44}

The varied structure and distribution of CD44 suggests that the molecule has a variety of functions. Known functions of CD44 are:

- cellular adhesion (aggregation and migration)

- hyaluronate degradation ${ }^{18}$

- lymphocyte activation ${ }^{33-37}$

- lymph node homing ${ }^{22} 38$

- myelopoiesis and lymphopoiesis ${ }^{39-41}$

- angiogenesis ${ }^{42}$

- release of cytokines ${ }^{43}$.

CD44 functions are principally dependant on cellular adhesion in one setting or another. ${ }^{44}$ This adhesion can lead to interaction between two different cells or between a cell and its pericellular matrix.

CELL-MATRIX INTERACTIONS INVOLVING CD44 In view of the variety of isoforms and the widespread tissue distribution of CD44 it is not surprising that this molecule has more than one ligand. These ligands include hyaluronate, chondroitin, collagen, laminin, and fibronectin. ${ }^{45}$ The main ligand for CD44 is hyaluronate. ${ }^{44}$ The amino-terminal (hyaluronan binding) domain is a constant, highly preserved feature of all CD44 isoforms. This terminal domain is composed of two clusters of positively charged amino acids, which bind a six-sugar sequence of hyaluronate. ${ }^{2146}$

Hyaluronate is a very long, high molecular weight polysaccharide molecule. It is distributed ubiquitously in the extracellular space of higher animals. Hyaluronate has other receptors such as the protein RHAMM (receptor for hyaluronic acid mediated motility).
The cell's ability to bind to hyaluronate via CD44 is finely regulated. ${ }^{47}{ }^{48}$ The clustering effect of CD44 on the cell surface has been shown to be necessary for the binding of hyaluronate because it is a multivalent interaction ${ }^{49}{ }^{50} \mathrm{An}$ intact cytoplasmic domain is also required for high affinity binding of hyaluronate. ${ }^{48}$ This binding affinity increases with molecular ionic strength because both molecules are negatively charged and binding is optimal at a neutral $\mathrm{pH} .^{51}$

The ability of variant isoforms of CD44 to bind hyaluronate is variable. ${ }^{52}{ }^{53}$ It appears that, although CD44v contains a hyaluronate binding domain, the molecule's ability to bind hyaluronate is reduced by the presence of its variant exons. ${ }^{53}$ If part of the variant domain is replaced by an alternative protein sequence the molecule's binding ability for hyaluronate increases. ${ }^{53}$ This inhibitory effect of the variant exons is additive, as a result of the degree of their glycosylation. Binding studies show that CD44v lacking O-linked glycosylation can bind hyaluronate as efficiently as CD $44 \mathrm{~s}^{53}$ $\mathrm{N}$-linked glycosylation has also been shown to reduce the ability of CD44 to bind hyaluronate. $^{545}$ This regulatory glycosylation can occur at the level of alternative splicing and also at the level of expression of glycosyltransferases. ${ }^{53}$

The CD44 molecule is also involved in the degradation of hyaluronate. The hyaluronateCD44 complex is internalised and then joined to a lysosomal compartment, where acid hydrolases act upon it. ${ }^{18}$ This is an important function of the CD44 molecule because build up of hyaluronate can have negative effects, such as in the lung, where it could interfere with gas exchange. ${ }^{18}$

Proliferating cells express high amounts of CD44. In these areas, hyaluronate concentrations are low and it has been suggested that 
localised degradation of hyaluronate leads to a morphological change in the tissue. ${ }^{18}$ Proliferating cells showing this expression include cells involved in the repair process, embryonic morphogenesis, organogenesis, and angiogenesis. ${ }^{183242}$ In addition, it was discovered that semi-purified fragments of hyaluronidase degraded hyaluronate could stimulate the proliferation and migration of endothelial cells and accelerate angiogenesis in vivo. ${ }^{56}{ }^{57}$ Interestingly, proliferating epithelial cells are typically rich in hyaluronate..$^{58}$

Smooth muscle cells involved in repair processes have been shown to upregulate both CD44 and the ligand, hyaluronate. Therefore, it has been suggested that smooth muscle cells establish an autocrine form of stimulation in the repair process. $^{59}$

Another CD44 ligand, chondroitin, is a polysaccharide and is closely related to hyaluronate. Chondroitin binds to the same region of the CD44 molecule as hyaluronate, the hyaluronan binding domain. Collagen, laminin, and fibronectin bind to CD44 by interaction with the chondroitin sulphate side chains that are linked to the core protein by glycosylation..$^{60}$

CELL-CELL INTERACTIONS INVOLVING CD44 CD44 is used by several cell types to mediate hyaluronate dependant adhesion. Aggregation of macrophages and lymphocytes is induced by exogenous hyaluronate. ${ }^{62}{ }^{63}$ Cultured fibroblasts can aggregate spontaneously by using endogenous hyaluronate present on the cells. ${ }^{64}{ }^{65}$ Hyaluronate based adhesion is reduced in the presence of hyaluronidase, low levels of hyaluronate, high concentrations of chondroitin, or monoclonal antibodies to the hyaluronate receptor. ${ }^{6366}$

CD44 binding of hyaluronate is relatively weak in comparison to other cell adhesion mechanisms, such as those involving cadherins or integrins. This has been suggested to be an advantage where cells only need to be held close together for the exchange of chemical signals. ${ }^{18}$ This could account for its properties of activation and maturation of lymphocytes. ${ }^{39}$

Exon v10 has a serine glycine motif that functions as a chondroitin sulphate attachment and is used in preference to the CD44 chondroitin/hyaluronate binding site. ${ }^{67}$ This moeity is recognised by other CD44 molecules and is bound. Exon v10 can promote homotypic and/or heterotypic cell-cell adhesion in vitro. ${ }^{67}$ Other exon specific attachments include exon v6, which has been shown to be a site of attachment in rats of the $\mathrm{H}$ blood group antigen. ${ }^{68}$ Glycosylation of exon v3 with heparan sulphate enables it to bind a subset of heparin binding growth factors, and cytokines including fibroblast growth factor $b$ (bFGF) and heparin binding epidermal growth factor (HB-EGF). ${ }^{69}$ This binding can act as a reservoir for these substances. ${ }^{70}$

More recent research has shown that another ligand for CD44, osteopontin (Eta-1; early T lymphocyte activation 1), is involved in CD44 dependent chemotaxis. ${ }^{71}$ Osteopontin has been shown to be involved in the regulation of inflammation, ${ }^{72}$ bone formation (osteogenesis) ${ }^{73}$ and angiogenesis, ${ }^{74}$ and this might depend on an interaction with CD44.

LYMPH NODE HOMING AND LYMPHOCYTE ACTIVATION

One of the main functions of CD44 is in lymph node homing ${ }^{2238}$ and lymphocyte activation. ${ }^{33}{ }^{34}$ Lymph node homing is achieved by a specific interaction between the middle domain of CD44s on lymphocytes and a protein, present on the high endothelial cells of Peyer's patches and lymph nodes, called mucosal addressin.

The variant CD44v6 has been shown to have a crucial role in the movement and homing of antigen activated lymphocytes in lymph nodes. ${ }^{36} \mathrm{CD} 44 \mathrm{v} 6$ is also required for activation of $\mathrm{B}$ and $\mathrm{T}$ lymphocytes and maturation of stem cell progenitors. ${ }^{36}$ The precise mechanisms involved have yet to be elucidated, although it is thought that activation and interaction of lymphocytes require crosslinking of CD44, and additional cofactors and adhesion molecules. The costimulatory signal of CD44 is thought to depend upon signalling through CD2. ${ }^{35}$ The variants CD $44 v 3$ to CD 44v6 have been shown to be expressed transiently as an early event on the blast cells of lymph nodes and the spleen after activation of lymphocytes. ${ }^{3637}$

\section{MYELOPOIESIS, LYMPHOPOIESIS, AND} ANGIOGENESIS

CD44 is also involved in both myelopoiesis and lymphopoiesis. ${ }^{39}$ CD44v6 is required for maturation of the adherent stem cell population in what may involve v6 primarily transducing signals between stromal cells and stem cells, which initiate differentiation. ${ }^{41}$ Upon ligand interaction, CD44s is thought to provide proliferation initiating signals for early progenitors of all three haematopoietic lineages. ${ }^{39}$

Proliferation and migration, two features required in angiogenesis, are inhibited when a monoclonal antibody to CD44 is used on calf pulmonary arterial endothelial cells. ${ }^{42}$

\section{Role of CD44 in neoplasia}

Tremendous interest in CD44 was generated when Günthert and colleagues ${ }^{2}$ conferred metastatic potential on a non-metastasising cell line by transfecting a variant of CD44. The initial work was to identify the membrane proteins on a metastasising rat pancreatic adenocarcinoma by the use of monoclonal antibodies. These antibodies were screened on a bacterial cDNA expression library and one of the cDNA clones was found to encode a variant isoform of CD44. This cDNA was transfected into a non-metastasising, rat pancreatic adenocarcinoma, which although expressing CD44s, did not express this particular variant. The new cell line was found to have gained metastatic properties when injected into rats (syngeneic animals). This phenomenon was also demonstrated in mammary carcinoma cell lines.

Guo and colleagues ${ }^{75}$ showed that monoclonal antibody specific for CD44s can 
completely inhibit the binding of cells of a human melanoma cell line (SMMU2) to hyaluronate in vitro and also inhibit the metastatic capability of the tumour cells in vivo. In addition, if the monoclonal antibody to CD44 was given seven days after tumour inoculation, the primary tumour continued to grow, although the tumour's metastatic capability was completely inhibited.

Hofmann and colleagues ${ }^{76}$ noted that the expression of CD44v by CREF (cloned rat embryonic fibroblasts) cells correlated with metastatic behaviour. In immunocompromised animals, the expression of low amounts of CD44v was sufficient to confer metastatic ability. CD 44v expression by these cells could be increased by transfecting the activated oncogene ras. It has been reported that further mutations are needed to account for the amount of overexpression by highly metastatic cell lines. ${ }^{76}$

In support of this initial experimental evidence implicating the involvement of CD44 in neoplasia, it has been seen that high amounts of CD44 are associated with several types of malignant tumours. These include carcinomas, high grade gliomas, and a number of nonHodgkin's lymphomas. ${ }^{177}$ High concentrations of hyaluronate have also been found in many tumours. ${ }^{78}$ The invasiveness of certain tumours has also been correlated with CD44 and hyaluronate concentrations. ${ }^{77-79}$

ROLE OF CD44 IN TUMORIGENESIS

There are many potential theories about the possible mechanisms involved with respect to the role of CD44 in tumorigenesis.

CD44 expression is associated with a high rate of cell division. The proliferation status of tumour cells increases when cultured on anti-v6 antibody coated plates. CD $44 \mathrm{v} 6$ on the cell surface is thought to crosslink with other CD44v6 molecules, initiating signals of growth promoting activity. ${ }^{41}$ Interactions between CD44 and its ligands might induce the tumour cells to produce autocrine growth factors. These factors might be critical for tumour growth. The functions of CD44 beyond cellular adhesion require the transmission of intracellular signals. Some of these signals are thought to occur via the cytoskeleton and might enable CD44 to signal to both the locomotory ${ }^{80} 42$ and mitogenic machinery of the cell. $^{42}$

Tumour cells expressing CD44 can adhere to the extracellular matrix through its ligands, including hyaluronan, chondroitin sulphate, fibronectin, laminin, collagen, and matrigel. This might allow tumour cells to colonise these environments more efficiently. The functions of uptake and degradation of hyaluronate by CD44 could enable tumour cells to invade hyaluronate rich tissues. In a panel of breast cancer cell lines, hyaluronate degradation mediated by CD44 correlated better than CD44 expression itself with invasiveness. ${ }^{81}$ Cytoplasmic staining of CD44 might be indicative of hyaluronate uptake and was found to be prevalent in a comprehensive panel of tumours. ${ }^{24}$ This cytoplasmic staining was also accentuated at the infiltrating tumour margin, which would be consistent with the proposed role of CD44 in tumour invasion. ${ }^{24}$ Tissue specific infiltration might require CD44v expression. This appears to be the case in infiltration of the skin, where CD $44 v 10$ is expressed by the infiltrating tumour. ${ }^{41}$

ROLE OF CD44 IN METASTASIS FORMATION

Metastatic spread requires a series of interactions between the tumour cells and the surrounding extracellular matrix and nontumour cells. These interactions will depend on cell surface determinants such as extracellular receptors for matrix and basal lamina, surface bound proteolytic enzymes, cell adhesion molecules, growth factors, and growth factor receptors. ${ }^{2}$ The metastasising tumour cell copies the same mechanisms of normal cellular migration. From the review of the physiological functions of CD44 it can be seen that CD44 can function as a cell surface determinant for several of the roles required for metastatic spread to occur.

The function of CD44v in the metastatic process can be blocked by a monoclonal antibody to CD44v. However, this observation does not reveal which step or steps CD44 is involved in, although the monoclonal antibody has to be given early on to block the metastatic process. $^{82} 83$

The theoretical steps of the metastatic process are known as the metastatic cascade ${ }^{84}$ and they consist of: (1) loss of contact with the surrounding tumour cells or neighbouring cells; (2) breakthrough of the basement membrane and penetration of vessel walls; (3) survival of shearing forces in the bloodstream/lymph stream; (4) adhesion and penetration through the vessel walls; (5) expansion into foreign tissue; (6) induction of vascularisation of tumour.

For a tumour cell to lose contact with neighbouring tumour cells, its adhesive properties must change. Changing the cell's CD44 profile could certainly achieve this. Increased expression of CD44 can enhance binding to hyaluronate and a pericellular matrix of hyaluronate might decrease the affinity of a cell for surrounding hyaluronate deficient cells by interfering with adhesion processes, thus leading to detachment. This increased mobility is thought to be initiated by CD44 because it is linked to the cell's cytoskeleton. ${ }^{42}{ }^{80}$ A CD44ligand complex could mediate the mechanical force and transmit intracellular locomotory signals via the cytoskeleton. This response could lead to the cells enhanced movement along hyaluronate rich surfaces. ${ }^{26}$ As previously discussed, CD44 has the ability to take up and degrade hyaluronate, ${ }^{18}$ and this property could allow tumour cells to escape entrapment within hyaluronate rich environments.

Migration of any cell to the vascular or lymphatic system requires both cell adhesion molecules and cell surface enzymes. The ability of CD44 to degrade hyaluronate could also be used by the tumour cell to assist in its path through the basement membrane and vessel wall. Tumour cells that metastasise by way of the lymphatic system are thought to imitate 
lymphocytes, entering peripheral lymphatics and travelling to the draining lymph nodes. ${ }^{36}$ Variants of CD44 are involved in the activation of lymphocytes and the release of cytokines. ${ }^{43}$ CD44s is required for lymphocyte homing within the lymphatics to the high endothelial venules within lymph nodes. ${ }^{22} 38$ In a lymphoma animal model, CD44 monoclonal antibodies did not inhibit spleen metastases developing, whereas they did affect the incidence of metastases to the lymph nodes. ${ }^{86}$ These observations imply that special variant isoforms of CD44 may be involved in the specific homing of tumour cells.

CD44 might also assist in distant metastatic adherence within the vascular system, with the adhesion molecule attaching to a known, or as yet unknown ligand. Tumours that produce a high concentration of hyaluronate might initiate aggregation through CD44s. This aggregate could become trapped in the capillary system as an embolus and invade through the vessel wall. CD44 also has a potential role in the next step of the metastatic cascade, which has been mentioned earlier in the role of CD 44 in tumorigenesis. This is the potential of CD44 to assist in the penetration through vessel walls and expansion into foreign tissue. Vascularisation of the newly embedded tumour may well involve CD44, which has been shown to be important in angiogenesis. ${ }^{42}$

\section{CD44 in specific tumour types}

Since the initial research showing the potentially crucial role of CD44 in tumorigenesis and the metastatic cascade, there have been many studies investigating the pattern of CD44 distribution in tumours.

Because CD44 is expressed in a wide variety of tissues, many studies have compared CD44 expression by particular tumours and their normal tissue counterpart. Tumours originating from epithelia that normally carry variants of CD44 on their surface remain positive for $\mathrm{CD} 44 \mathrm{v}$ and often produce higher concentrations of CD44 transcripts. ${ }^{3187}$ However, this finding is not uniform. Hudson and colleagues $^{88}$ found that squamous cell carcinomas arising from stratified squamous epithelium had a reduced expression of one or more of the variant exons that are expressed normally. This reduced expression of CD44 variants was greatest in the least differentiated regions and in distant metastases. Squamous cell carcinomas also showed changes in expression of other adhesion molecules, such as integrins.

Certain observations suggest that some cells do not use CD44 in tumorigenesis or in the production of metastases. In neuroblastoma, Burkitt's lymphoma, and melanoma CD44 variants are often absent. ${ }^{80}$ In one study, absence of CD44s correlated with poor survival in patients with neuroblastoma. ${ }^{90}$ In another study, transfection of CD44s into a highly metastatic rat prostate carcinoma cell line showed that this molecule acted as a metastasis suppressor gene, ${ }^{91}$ although the in vivo growth rate and tumorigenicity of the cell line were not suppressed.
CD44 EXPRESSION IN LUNG CARCINOMA

Givehchian and colleagues ${ }^{92}$ discovered that squamous cell carcinomas arising from the lung retain the CD44 expression profile of the normal respiratory epithelium, whereas all other types of lung cancer showed a significantly reduced, or absent, expression of either standard or variant CD44. These experiments did not provide any evidence for the presence of tumour or metastasis associated CD44 transcripts with an altered exon composition compared with CD44 transcripts expressed in the normal respiratory epithelium. The findings in the non-squamous cell carcinomas also suggested that downregulation of CD44s and CD $44 \mathrm{v}$ might contribute to metastatic spread, rather than enhanced expression of specific variant CD44 isoforms.

CD44 EXPRESSION IN GASTROINTESTINAL CARCINOMAS

An interesting finding by Higashikawa and colleagues $^{93}$ was the expression of a CD44 intron by colorectal tumours. In nonmalignant tissues, the intron 9 sequence is found in squamous epithelia and to a lesser degree, transitional and respiratory epithelia. Overexpression of variants containing intron 9 was also found frequently in intestinal metaplasia. The authors suggested that intron expression might be a tumour specific abnormality, except possibly in squamous epithelia. If tumour progression follows physiological genetic programmes, the possibility that colorectal tumours are using a variant of CD44 that serves a physiological function in squamous epithelium must be considered.

Higashikawa and colleagues ${ }^{93}$ also found that all the colorectal adenocarcinomas they examined overexpressed high molecular weight CD44 transcripts including the v6 exon. The CD44v6 variant was not found to correlate with tumour stage or metastatic status and was suggested to be a common phenotype of colorectal cancer. However, some studies have found the CD44v9 variant to be an independent prognostic indicator for gastrointestinal tumours. Mayer and cooleagues ${ }^{94}$ found that CD44v9 expression in primary gastric cancers had a statistical correlation with tumour recurrence and mortality. Yamaguchi and colleagues $^{95}$ found that the rate of CD44v8v10 expression was significantly higher in tumours from patients with liver metastases than those without, but the authors could find no correlation between CD44v8-v10 expression and prognosis. However, in a later paper, Yamaguchi and colleagues ${ }^{96}$ found CD44v8v10 expression to be an independent prognostic indicator for colorectal carcinoma. Takahashi and colleagues ${ }^{97}$ have found that expression of CD44s by colon carcinoma cells enhances their hyaluronate binding, and this enhancement appears to reduce both in vitro and in vivo growth of the carcinoma cells. The authors also report that the hyaluronate binding activity of CD44 was less pronounced in highly metastatic cell lines compared with poorly metastatic cells lines. The differences in hyaluronate binding ability were suggested to 
be caused by post-translational modifications. These observations led to the conclusion that the functional consequences of the association of decreased CD44s expression with colonic mucosa transformation might be just as important as increased expression of high molecular weight variants of CD44 by tumours.

Different types of gastric cancer have different patterns of CD44 expression. The intestinal type of gastric cancer has a predominant expression of CD44v6, whereas the diffuse type is mostly CD44v6 negative. ${ }^{98}$ It is interesting that the CD44v6 positive intestinal type appeared to have a more infiltrative type of growth compared with the CD44v6 negative diffuse type. Müller and colleagues ${ }^{99}$ found no correlation between C44v6 expression in gastric carcinoma and prognosis. CD44v5 expression correlated with significantly shorter overall survival times, although this significance was lost with the use of multivariate analysis.

CD44 EXPRESSION IN MALIGNANT MELANOMA Research with melanoma cell lines has revealed that transfection of a CD44 negative melanoma cell line with cDNA for the CD44s molecule promotes tumorigenicity. ${ }^{100}$ Further work showed that growth and metastasis of melanoma cells can be inhibited by monoclonal antibodies to $\mathrm{CD} 44,{ }^{75}$ and that the degree of CD44 expression correlates with the size of the pulmonary metastases produced. ${ }^{101}$ In addition, lymph node metastases had a significantly higher expression of CD44 antigen when compared with the primary tumour. ${ }^{30}$ It is interesting that melanocytes and naevi do not express CD44 within an epithelium which does express the molecule, and that melanomas appear to require CD44 for tumorigenicity. Also epithelia are, obviously, matrix free.

Danen and colleagues ${ }^{102}$ reported that uveal melanomas that arise from retinal epithelium appear to express a different CD44 pattern when compared with cutaneous melanomas. CD44v6 tends to be expressed by in situ uveal melanomas and not cutaneous melanomas and vice versa for CD44v5. The authors suggest that the microenvironment of the melanoma cells influences expression of this variant.

CD44 EXPRESSION IN BREAST CARCINOMA

Studies looking into CD44 expression in breast carcinomas have indicated that expression could be regulated by hormones. Increases in the expression of oestrogen and progesterone receptors showed a positive correlation with CD44v6. ${ }^{103}$ Several studies have evaluated CD44 expression in breast carcinomas with conflicting results. Sinn and colleagues ${ }^{104}$ found a correlation between expression of CD44v3/v4 and CD44v6 and increased tumour grade, whereas Friedrichs and colleagues $^{103}$ found a correlation between expression of CD44s and CD44v9 and tumour grade. Neither study found CD44 or any of its variants to be independent prognostic indicators, as was the case in a similar study by Kaufmann et al. ${ }^{105}$ Differences between studies might result from varying techniques of recog- nition. Using immunohistochemistry, Sinn and colleagues ${ }^{104}$ showed a higher expression of CD44 at the tumour-stroma interface and that all regional lymph node metastases were homogeneously positive for variants of CD44. A more recent study, ${ }^{107}$ using immunohistochemistry on 218 primary breast carcinomas, found that CD44s and CD44v6 did not correlate with known predictors of poor prognosis.

CD44 EXPRESSION IN PROSTATE CARCINOMA

Preliminary studies on carcinoma of the prostate indicate altered expression of CD44 in these tumours. ${ }^{107}$

CD44 EXPRESSION IN LEUKAEMIA

Rossi and colleagues ${ }^{108}$ found that CD44s was overexpressed in approximately half their cases of B cell chronic lymphocytic leukaemia, although CD44v5 and CD44v6 expression was usually found to be normal. Raised serum CD44s concentrations were associated with a number of unfavourable prognostic factors and the authors proposed that the molecule might be a reliable tumour marker.

CD44 EXPRESSION IN PANCREATIC CARCINOMA A study of pancreatic carcinomas and CD44 expression ${ }^{109}$ revealed that the variant CD44v6 is found in normal ductal cells as well as in cancer specimens. Using reverse transcription PCR (RT-PCR), the study found a difference between the combinations of exon expression. In normal ductal cells, CD44v6 tends to be the only splice product, whereas in carcinomas the CD44v6 exon was associated with severa1 other variant exons, leading to an overexpression of multiple splice variants. The authors suggest that the chain composition of the CD44 variant chains might be important for their altered function. Satoh and colleagues ${ }^{110}$ discovered that the variant exons v5 and v6 were expressed by almost all duct cell carcinomas and infrequently expressed by the more benign intraductal carcinomas. CD44s was found to be frequently expressed by both types of pancreatic carcinoma.

CD44 EXPRESSION IN THYROID CARCINOMA Ermak and colleagues ${ }^{111}$ showed that papillary cancers of the thyroid also appear to follow certain restricted patterns of CD44 variant expression. Several CD44 isoforms are not detectable in normal thyroid tissue and CD44v6 and CD44v7 are coexpressed in a tightly coupled manner in these thyroid cancers.

CD44 EXPRESSION IN OVARIAN CARCINOMA Sliutz and colleagues ${ }^{112}$ showed that ovarian tumour samples exhibit a more complex pattern of CD44 expression than normal ovarian tissue, and proposed that this expression is reflected in its serum concentration. However, the variants of CD44 detected in the serum did not correlate with any clinical parameter, and it was suggested that the dominant serum CD44 variants were produced by natural sources masking the pathological output. 
CD44 EXPRESSION IN SARCOMAS

There has been comparatively little research done on the expression of CD44 by sarcomas. Wang and colleagues ${ }^{113}$ discovered that most sarcomas express CD44s. Certain sarcomas appeared to have a higher incidence of CD44s expression (epithelioid sarcoma and angiosarcoma) compared with others (clear cell sarcoma and liposarcoma). Immunohistochemical staining of the cytoplasm was also reported, which might represent hyaluronate uptake. ${ }^{24}$ No correlations between CD44s positivity and clinical outcome could be made because of the relatively small numbers. Our own research ${ }^{114}$ has shown that there is no correlation between CD44s or variant isoform expression and clinical outcome in synovial sarcomas.

In summary, the above studies have produced wildly conflicting data. Most papers suggest that this results from a lack of consistency in experimental method. Some studies rely on PCR analysis whereas others generate data from immunohistochemical analysis. Different monoclonal antibodies have been used to detect the presence of the same isoform, some antibodies being more sensitive than others.

\section{Conclusion}

CD44 is a ubiquitous transmembrane cell surface molecule. Its principal physiological functions are in the aggregation, migration, and activation of cells, and these occur through the adhesive qualities of the molecule. CD44 is overexpressed by many tumours, and the pattern of expression often differs from that of the tissue of origin. This feature might be useful in screening these tumours using PCR analysis. CD44 is able to endow certain non-metastatic cell lines with metastatic properties and this effect can be blocked by monoclonal antibodies to CD44. CD44 possesses a number of properties that are required by a metastasising cell. Expression of CD44 and variants of CD44 have been correlated with clinical outcome in several human malignancies, although these findings are not consistent between various published studies. Further studies are required with standardisation of methodologies, larger study samples, and longer clinical follow up periods to establish the prognostic significance of CD44 and its variant isoforms.

The precise function of CD44 in the metastatic process and the degree of involvement in human malignancies has yet to be established fully.

1 Stamenkovic I, Amiot M, Pesando J, et al. A lymphocyte molecule implicated in lymph node homing is a member of the cartilage link protein family. Cell 1989;56:1057-62.

2 Günthert U, Hofmann M, Rudy W, et al. A new variant of glycoprotein CD44 confers metastastic potential to rat carcinoma cells. Cell 1991;65:13-24.

3 Hughes E, Mencod G, Aucrust T. Murine cell surface glycoproteins. Characterization of a major component of 80,000 daltons as a polymorphic differentiation antigen of mesenchymal cells. f Biol Chem 1981;256:7023-7.

4 Trowbridge I, Lesley J, Schulte R, et al. Biochemical characterisation and cellular distribution of a polymorphic murine cell surface glycoprotein expressed on lymphoid murine cell surface glycoprotein expressec

5 Carter W, Wayner E. Characterisation of the class III collagen receptor, a phosphorylated transmembrane glycopro-
tein expressed in nucleated human cells. $\mathcal{F}$ Biol Chem 1988 ; 263:4193-201.
6 Gallatin W, Wayner E, Hoffman P, et al. Structural homology between lymphocyte receptors for high endothehomology between lymphocyte receptors for high endotheAcad Sci USA 1989;86:46548.

7 Jalkanen S, Bargatze R, Herron L, et al. A lymphoid cell surface protein involved in endothelial recognition and ymphocyte homing in man. Eur F Immunol 1986;16:1195202.

8 Haynes B, Telen M, Hale L, et al. CD44-a molecule involved in leukocyte adherance and T-cell activation. Immunol Today 1989;10:423-7.

9 Haynes B, Liao H, Patton K. The transmembrane hyaluronate receptor (CD44); multiple functions, multiple forms. Cancer Cells 1991;3:347-50.

10 Goodfellow PN, Banting G, Wiles MV, et al. The gene, MIC4, which controls expression of the antigen defined by MIC4, which controls expression of the antigen defined by 11. Eur F Immunol 1982;12:659-63.

11 Hofmann M, Rudy W, Zoller M, et al. CD44 splice variants confer metastatic behaviour in rats: homologous sequences are expressed in human tumour cell lines. Cancer Res 1991; 51:5292-7.

12 Gallagher T. The protein and proteoglycan guises of Hermes/CD44. Glycobiology 1992;2:93-7.

13 van Weering D, Baas P, Bos J. A PCR-based method for the analysis of human CD44 splice products. PCR Methods Appl 1994;3:100-6.

14 Hardingham T, Fosang A. Proteoglycans: many forms and many functions. FASEB $\mathcal{f} 1992 ; 6: 861-70$

15 Brown T, Buouchard T, St John T, et al. Human keratinocytes express a new CD44 core protein as a heparan-sulfate intrinsic membrane proteoglycan with additional exons. $\mathcal{F}$ Cell Biol 1991;113:207-21.

16 Idzerda R, Carter W, Nottenburg C, et al. Isolation and DNA sequence of a cDNA clone encoding a lymphocyte adhesion receptor for high endothelium. Proc Natl Acad Sci USA 1989;86:4659-63.

17 Goldstein L, Butcher E. Identification of mRNA that encodes an alternative form of H-CAM (CD44) in ymphoid and nonlymphoid tissues. Immunogenetics 1990; 32:389-97.

18 Underhill C. CD44: the hyaluronan receptor. $f$ Cell Sci 1992;103:293-8.

19 Neame J, Isacke $M$. Phosphorylation of CD44 in vivo requires both Ser323 and Ser325, but does not regulate requires both Ser323 and Ser325, but does not regulate membrane localization or cytoskeleton

20 Neame J, Isacke $M$. The cytoplasmic tail of CD44 is required for basolateral localization in epithelial MDCK cells but does not mediate association with the detergentinsoluble cytoskeleton of fibroblasts. F Cell Biol 1993;121: 1299-310.

21 Yang B, Yang BL, Savani RC, et al. Identification of a common hyaluronan binding motif in the hyaluronan binding proteins RHAMM, CD 44 and link protein. EMBO 71994 13:286-96.

22 Jalkanen S, Bargatze R, Herron L, et al. A lymphoid cell surface glycoprotein involved in endothelial cell recognition face glycoprotein involved in endothelial cell recognition and lymph 202 .

23 Cooper D, Dougherty G, Harn H, et al. The complex CD44 transcriptional unit: alternative splicing of three internal exons generates the epithelial form of CD44. Biochem Biophys Res Commun 1992;182:569-78.

24 Fox S, Fawcett J, Jackson D, et al. Normal human tissues, in addition to some tumors, express multiple different CD44 isoforms. Cancer Res 1994;54:4539-46.

25 Mackay C, Terpe H-J, Stauder R, et al. Expression and modulation of CD44 variant isoforms in humans. $f$ Cell Biol 1994;124:71-82.

26 Sleeman J, Moll J, Sherman L, et al. The role of CD44 slice variants in human metastatic cancer. In: Cell adhesion and variants in human metastatic cancer. In: Cell adh

27 Fox S, Gatter K, Jackson D, et al. CD44 and cancer screening. Lancet 1993;342:548-9.

28 Kugelman L, Ganguly S, Haggerty J, et al. The core protein of epican, a heparan sulfate proteoglycan on keratinocytes, is an alternative form of CD44. F Invest Dermatol 1992;99: $381-5$.

29 Dall P, Hekele A, Ikenberg H, et al. Increasing incidence of $\mathrm{CD} 44 \mathrm{v} 7 / 8$ epitope expression during uterine cervical carcinogenesis. Int f Cancer 1996;69:79-85.

30 Korabiowska M, Brinck U, Hönig J, et al. CD-44: a marker of metastases of head and neck melanomas. In Vivo 1995; 9:253-6.

31 Wirth K, Arch R, Somasundaram C, et al. Expression of CD44 isoforms carrying metastasis-associated seqences in newborn and adult rats. Eur $\mathcal{F}$ Cancer 1993;29A: $1172-7$.

$32 \mathrm{Yu}$ Q, Toole B. Common pattern of CD44 isoforms is expressed in morphogenetically active epithelia. Dev Dyn 1997;208:1-10

33 Shimizu Y, Seventer van G, Siraganian R, et al. Dual role of the CD44 molecule in T-cell adhesion and activation. $f$ Immunol 1989;143:2457-63.

34 Huet S, Groux H, Caillou B, et al. CD44 contributes to T-cell activation. F Immunol 1989;143:798-801.

35 Conrad P, Rothman B, Kelley K, et al. Mechanism of peripheral $\mathrm{T}$ cell activation by coengagement of CD 44 and peripheral T cell activation by coen

36 Arch R, Wirth, K, Hofmann $M$, et al. Participation in normal immune responses of a metastasis-inducing splice variant of CD44. Science 1992;257:682-5. 
37 Koopman G, Heider K-H, Horst E, et al. Activated human lymphocytes and aggressive non-Hodgkin lymphomas express a homologue of the rat metastasis-
variant of CD44. Exp Med 1993;177:897-904.

38 Jalkanen S, Bargatze R, DeLosToyes J, et al. Lymphocyte recognition of high endothelium: antibodies to distinct epitopes of an $85-95 \mathrm{kD}$ glycoprotein antigen differentially inhibit lymphocyte binding to lymph node, mucosal, or synovial endothelial cells. $\mathcal{F}$ Cell Biol 1987;105:983-90. 39 Miyake K, Medina K, Hayashi S, et al. Monoclonal antibodbone marrow cultures. $\mathcal{F}$ Exp Med 1990;171:477-88

40 Kincade P. Molecular interactions between stromal cells and B lymphocyte precursors. Semin Immunol 1991;3:379-90.

41 Zöller M. CD44: physiological expression of distinct isoforms as evidence for organ-specific metastasis formaisoforms as evidence for organ-sped
tion. $\mathcal{F ~ M o l ~ M e d ~ 1 9 9 5 ; 7 3 : 4 2 5 - 3 8 . ~}$

42 Trochon V, Mabilat C, Bertrand P, et al. Evidence of involvement of CD44 in endothelial cell proliferation, migration and angiogenesis in vitro. Int $\mathcal{F}$ Cancer 1996;66: migration

43 Webb D Shimizu Y, Seventer van G, et al. LFA-3, CD44, and CD45: physiological triggers of human monocyte TNF and IL-1 release. Science 990;249:1295-7.

44 Aruffo A, Stamenkovic 1, Melnick M, et al. CD44 is the principal cell surface receptor for hyaluronate. Cell 1990;61:1303-13.

45 Wayer E, Carter W. Identification of multiple cell adhesion receptors for collagen and fibronectin in human fibrosarcoma cells possessing unique alpha and common beta subunits. F Cell Biol 1987;105:1873-84.

46 Peach R, Hollenbaugh D, Stamenkovic I, et al. Identification of hyaluronic acid binding sites in the extracellular domain of CD44. F Cell Biol 1993;122:257-64.

47 Lesley J, Schulte R, Hyman R. Binding of hyaluronic acid to lymphoid cell lines is inhibited by monoclonal antibodies lymphoid cell lines is inhibited by monoclon
against Pgp-1. Exp Cell Res 1990;187:224-33.

48 Lesley J, He Q, Miyake K, et al. Requirements for hyaluronic acid binding by CD44: a role for the cytoplasmic domain and activation by antibody. $\mathcal{F}$ Exp Med 1992;175:257-66.

49 Underhill C, Toole B. Physical characteristics of hyaluronate binding to the surface of simian virus 40 transformed 3T3 cells. F Biol Chem 1980;255:4544-9.

50 Lesley J, Kincade P, Hyman R. Antibody-induced activation of the hyaluronan receptor function of CD44 requires multivalent binding of antibody. Eur F Immunol 1993;23:19029.

51 Underhill C. Interaction of hyaluronate with the surface of simian virus 40-transformed 3T3 cells: aggregation and binding studies. F Cell Sci 1982;56:177-89.

52 Stamenkovic I, Aruffo A, Amiot M, et al. The haematopoietic and epithelial forms of CD44 are distinct polypeptides etic and epithelial forms of CD 44 are distinct polypeptides with different adhesion potential

53 Bennett K, Modrell B, Greenfield B, et al. Regulation of CD44 binding to hyaluronan by glycosylation of variable spliced exons. F Cell Biol 1995;131:1623-33.

54 Katoh S, Zheng Z, Oritani K, et al. Glycosylation of CD44 negatively regulates its recognition of hyaluronan. $\mathcal{F} \operatorname{Exp}$ Med 1995;182:419-29.

55 Lesley J, Hyman R. CD44 can be activated to function as an hyaluronic acid receptor in normal murine T cells. Eur $\mathcal{F}$ Immunol 1992;22:2719-23.

56 West D, Kumar S. The effect of hyaluronate and its oligosaccharides on endothelial cell proliferation and monolayer integrity. Exp Cell Res 1989;183:179-96.

57 Lees V, Fan T, West D. Angiogenesis in a delayed revascularization model is accelerated by angiogenic revascularization model is accelerated by angiogenic

58 Alho A, Underhill C. The hyaluronate receptor is preferentially expressed on proliferating epithelial cells. $\mathcal{f}$ Cell Biol

59 Jain M, He Q, Lee W, et al. Role of CD44 in the reaction of vascular smooth muscle cells to arterial wall injury. $\mathcal{F}$ Clin Invest 1996;97:596-603.

60 Faassen A, Schrager J, Klien D, et al. A cell surface chondroitin sulfate proteoglycan, immunologically related to $\mathrm{CD} 44$, is involved in type 1 collagen-mediated melanom cell motility and invasion. f Cell Biol 1992;116:521-31.

61 Jalkanen S, Jalkanen M, Bargatze R, et al. Biochemical properties of glycoproteins involved in lymphocyte recognition of high endothelial venules in man. F Immunol 1988;141:

62 Pessac B, Defendi V. Cell aggregation: role of acid mucopolysaccharides. Science 1972;175:898-900.

63 Green S, Tarone G, Underhill C. Aggregation of macrophages and fibroblasts is inhibited by a monoclonal. antibody to the

64 Underhill C, Dorfman A. The role of hyaluronic acid in intercellular adhesion of cultured mouse cells. Exp Cell Res 1978;117:155-64.

65 Underhill C, Toole B. Receptors for hyaluronate on the surface of parent and virus-transformed cell lines: binding and aggregation studies. Exp Cell Res 1981;131:419-23.

66 Underhill C, Green S, Comoglio P, et al. The hyaluronate receptor is identical to a glycoprotein of $85,000 \mathrm{Mr}$ (gp85) as shown by a monoclonal antibody that interferes with binding activity. $\mathcal{F}$ Biol Chem 1987;262:13142-6.

67 Cooper D, Doughtery G. To metastasize or not? Selection of CD44 splice sites. Nat Med 1995;1:635-7.

68 Labarriere N, Pian J, Otry C, et al. $\mathrm{H}$ blood group antigen carried by CD $44 \mathrm{~V}$ modulates tumorigenicity of rat colon carcinoma cells. Cancer Res 1994;54:6275-81.
69 Bennett K, Jackson D, Simon J, et al. CD44 isoforms containing exon $\mathrm{v} 3$ are responsible for the presentation of containing exon v3 are responsible for the presentation of

70 Yanagishita $M$, Hascall V. Cell surface heparan sulfate proteoglycans. F Biol Chem 1992;267:9451-4.

71 Weber G, Ashkar S, Glimcher $M$, et al. Receptor-ligand interaction between CD44 and osteopontin (Eta-1). Science 1996;271:509-12.

72 Singh R, Patarca R, Schwartz J, et al. Definition of specific interaction between early-T-lymphocyte-activation-1 (eta-1) protein and murine macrophages in vitro and in vivo. f Exp Med 1990;171:1931-42.

73 Reinholt F, Hultenby K, Oldberg A, et al. Osteopontin-a possible anchor of osteoblasts to bone. Proc Natl Acad Sci USA 1990;87:4473-5.

74 Yue T, McKenna P, Ohlstein E, et al. Osteopontinstimulated vascular smooth muscle cell is mediated by beta 3 integrin. Exp Cell Res 1994;214:459-64.

75 Guo Y, Ma J, Wang J, et al. Inhibition of human melanoma growth and metastasis in vivo by anti-CD44 monoclonal antibody. Cancer Res 1994;54:1561-5.

76 Hofmann M, Rudy W, Günthert U, et al. A link between ras and metastatic behaviour of tumor cells: ras induces CD44 promoter activity and leads to low-level expression of metastases-specific variants of CD44 in CREF cells. Cancer Res 1993;53:1516-21.

77 Horst E, Meijer C, Radaszkiewicz T, et al. Adhesion molecules in the prognosis of diffuse large-cell lymphoma: expression of a lymphocyte homing receptor (CD44), 1990;4:595-9.

78 Zigmund S. Cell locomotion and chemotaxis. Curr Opin Cell Biol 1989;1:80-6.

79 Namec R, Toole B, Knudson T. The cell surface hyaluronate binding sites of invasive human bladder carcinoma cells. Biochem Biophys Res Commun 1987;149:249-57.

80 Bourguignon L, Lokeshwar V, Chen X, et al. Hyaluronic acid induced lymphocyte signal transduction and HA receptor (GP85/CD44)-cytoskeleton interaction. f Immunol 1993;151:6634-44.

81 Culty M, Shirazi M, Thompson E, et al. Binding and degradation of hyaluronan by human breast cancer cell lines expressing different forms of CD44: correlation with invasive potential. F Cell Physiol 1994;160:275-86.

82 Reber S, Matzku S, Güthert U, et al. Retardation of metastatic tumor growth after immunisation with metastasis-specific monoclonal antibodies. Int $\mathcal{f}$ Cancer 1990;46:919-2 7 . 83 Seiter S, Arch R, Reber S, et al. Prevention of tumor metas-
tasis formation by anti-variant CD 44. $\mathcal{F}$ Exp Med 1993;177:443-55.

84 Jiang W. In vitro models of cancer invasion and metastasis: recent developments. Eur f Surg Oncol 1994;20:493-9.

85 Zetter B. Adhesion molecules in tumor metastasis. Semin Cancer Biol 1993;4:219-29.

86 Zahalka M, Okon E, Gosslar U, et al. Lymph node (but not spleen) invasion by murine lymphoma is both CD44- and spleen) invasion by murine lymphoma is both CD44-
hyaluronate-dependent. F Immunol 1995;154:5345-55.

87 Heider K, Hofmann M, Horst E, et al. A human homologue of the rat metastasis-associated variant of CD44 is expressed in colorectal carcinomas and adenomatous polyps. F Cell Biol 1993;120:227-33.

88 Hudson D, Speight P, Watt F. Altered expression of CD44 isoforms in squamous-cell carcinomas and cell lines derived from them. Int f Cancer 1996;66:457-63.

89 Salmi M, Grön-Virta K, Sointu P, et al. Regulated expression of exon v6 containing isoforms of CD44 in man: downregulation during malignant transformation of tumors of squamocellular origin. $\mathcal{F}$ Cell Biol 1993;122:431-42.

90 Favrot M, Combaret V, Lasset C, et al. CD44-a new prognostic marker for neuroblastoma. N Engl f Med 1993;329: 196.

91 Gao A, Lou W, Dong J-T, et al. CD44 is a metastasis suppressor gene for prostatic cancer located on human Res 1997:57:846-9.

92 Givehchian M, Woerner S, Lacroix J, et al. Expression of CD44 splice variants in normal respiratory epithelium and 11 bronchial carcinomas: no evidence for altered CD44 splicing in metastasis. Oncogene 1996;12:1137-44.

93 Higashikawa K, Yokozaki $\mathrm{H}$, Ue T, et al. Evaluation of CD44 transcription variants in human digestive tract carcinomas and normal tissues. Int $\mathcal{F}$ Cancer 1996;66:11-17.

94 Mayer B, Jauch K, Günthert U, et al. De-novo expression of $\mathrm{CD} 44$ and

95 Yamaguchi A, Saito M, Goi T, et al. Expression of CD44 variant exons $8-10$ in gastric cancer. $\mathcal{F p n} \mathcal{f}$ Cancer Res 1995;86:1166-71.

96 Yamaguchi A, Urano T, Goi T, et al. Expression of a CD44 variant containing exons 8 to 10 is a useful independent factor for the prediction of prognosis in colorectal cancer patients. F Clin Oncol 1996;14:1122-7.

97 Takahashi. K, Stamenkovic I, Cutler M, et al. CD44 hyaluronate binding influences growth kinetics and tumorigenicity of human colon carcinomas. Oncogene 1995;11: 2223-32.

98 Dämmrich J, Vollmers H, Heider K, et al. Importance of different CD44v6 expression in human gastric intestinal and diffuse type cancers for metastatic lymphogenic spreading. f Mol Med 1995;73:395-401.

99 Müller W, Schneiders A, Heider K-H, et al. Expression and prognostic value of the CD44 splicing variants vS and v6 in gastric cancer. $\mathcal{F}$ Pathol 1997;183:222-7. 
100 Bartolazzi A, Peach R, Aruffo A, et al. Interaction between $\mathrm{CD} 44$ and hyaluronate is directly implicated in the regula-

101 Birch M, Mitchell S, Hart J. Isolation and characterisation of human melanoma cell variants expressing high and low of human melanoma cell variants expressing

102 Danen E, Berge P, Muijen G et al. Expression of CD44 and the pattern of CD44 alternative splicing in uveal melanoma. Melanoma Res 1996;6:31-5.

103 Friedrichs K, Franke F, Lisboa B-W, et al. CD44 isoform correlate with cellular differentiation but not prognosis in human breast cancer. Cancer Res 1995;55:5424-33.

104 Sinn H-P, Heider K-H, Skroch-Angel P, et al. Human mammary carcinomas express homologues of rat metastasis-associated variants of CD44. Breast Cancer Res Treat 1995;36:307-13.

105 Kaufmann M, Heider K-H, Sinn H-P, et al. CD44 variant exon epitopes in primary breast cancer and length of

106 Charpin C, Garcia S, Bouvier C, et al. Automated and quantitative immunocytochemical assays of CD44v6 in quantitative immunocytochemical assays of CD
breast carcinomas. Hum Pathol 1997;28:289-96.

107 Stevens J, Palechek P, Griebling T, et al. Expression of CD44 isoforms in human prostate tumor cell lines. Prostate 1996;28:153-61.
108 Rossi G, Marroni P, Paganuzzi M, et al. Increased serum levels of soluble CD44 standard, but not of variant soforms vS and v6, in B cell chronic lymphocytic leukemia. Leukemia 1997;11:134-41.

109 Gansauge F, Gansauge S, Zobywalski A, et al. Differential expression of CD44 splice variants in human pancreatic enocarcinoma and in the normal pancrea 1995;55:5499-503.

110 Satoh K, Shimosegawa T, Koizumi M, et al. Expression of CD44 in duct cell carcinomas and in intraductal neoplasms of the pancreas. Anticancer Res 1997;17:215-20.

111 Ermak G, Jennings T, Robinson L, et al. Restricted patterns of CD44 variant expression in human papillary thyroid carcinoma. Cancer Res 1996;56:1037-42.

112 Sliutz G, Tempfer C, Winkler S, et al. Immunohistochemical and serological evaluation of CD44 splice variants in human ovarian cancer. Br F Cancer 1995;72:1494-7.

113 Wang H, DeYoung B, Swanson P, et al. CD44 immunoreactivity in soft-tissue sarcomas. Applied Immunohistochemistry 1996;4:184-9.

114 Sneath RJ, Mangham D, Sneath R. The pattern of CD44 expression and clinical outcome of synovial sarcomas. Acta Orthop Scand 1997;68(suppl 276):22. 